\title{
20 years of nanotube transistors
}

\author{
Researchers in the field of carbon nanotube electronics continue to explore the potential of both high-performance \\ and thin-film transistors.
}

-

ield-effect transistors made from carbon nanotubes were first reported 20 years ago. In May 1998, and writing in Nature, Cees Dekker and colleagues at Delft University of Technology described using a single semiconducting single-walled carbon nanotube to build a transistor that could operate at room temperature ${ }^{1}$. Then, in October 1998, and writing in Applied Physics Letters, Phaedon Avouris and colleagues at IBM's T. J. Watson Research Center in New York described building transistors from single- and multi-walled carbon nanotubes ${ }^{2}$. This was only seven years after Sumio Iijima of the NEC Corporation had awakened interest in these rolled-up sheets of carbon atoms with a report on the synthesis of multiwalled carbon nanotubes ${ }^{3}$ and five years after reports on the growth of single-walled carbon nanotubes had appeared ${ }^{4,5}$.

The unique properties of carbon nanotubes, such as their high carrier mobility, suggested that the material could be of immense value in electronic devices, and expectations grew. But the field faced many technical challenges, not least the fact that nanotubes come in various structures and sizes, and can be both metallic and semiconducting, whereas high-performance devices demand samples with little structural variation. And the rate of progress was slower than many expected. (In 2002, for example, Avouris told the The New York Times that it might be ten years before nanotube chips were commercially available ${ }^{6}$.) The emergence of graphene, and other two-dimensional materials, also diverted the attention of many researchers, and wider scientific and technological interest in nanotube electronics wavered.

Nevertheless, the last two decades have seen impressive developments in the field and a variety of technology milestones have been passed. Logic circuits made from nanotube transistors were, for example, reported in $2001^{7}$; a five-stage ring oscillator with a stage delay of $2 \mathrm{~ns}$ was built on a single nanotube in $2006^{8}$; and a nanotube computer was created in $2013^{9}$, a system that has similar capabilities to the first commercial microprocessor, the Intel 4004

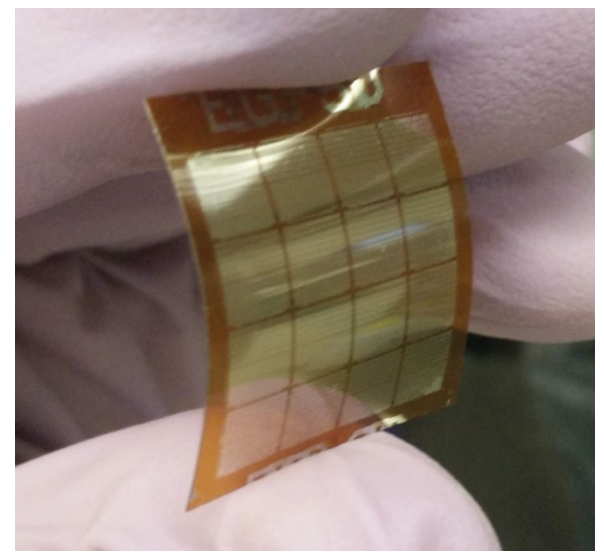

Carbon nanotube thin-film transistors fabricated on a flexible substrate. Credit: adapted from ref. ${ }^{15}$, Macmillan Publishers Ltd.

(ref. ${ }^{10}$ ), which was released in 1971 . More recently, notable advances in transistor scaling have been made, leading to nanotube transistors with a gate length of $5 \mathrm{~nm}$ (ref. ${ }^{11}$ ) and a footprint of $40 \mathrm{~nm}$ (ref. ${ }^{12}$ ). There have also been improvements in integrated circuit performance, leading to nanotube ring oscillators with encouraging performance $\mathrm{e}^{13,14}$.

Such work on high-performance transistors is focused on the grand goal of replacing silicon-based complementary metal-oxide-semiconductor (CMOS) technology, and is designed for logic applications on rigid substrates. But nanotubes, with their combination of high mobility and mechanical flexibility, are also well-suited to use in thin-film transistors (TFTs) on flexible substrates, where requirements in terms of performance and scaling are more relaxed. These flexible devices have an array of potential applications, including wearable devices and the Internet of Things (IoT), and have been a focus for many nanotube researchers in recent years.

The performance of flexible nanotube circuits is typically significantly lower than those built on rigid substrates. Nanotubebased flexible ring oscillators, for example, usually have stage delays longer than $1 \mu$ s. In this issue of Nature Electronics, Jianshi Tang,
Shu-Jen Han and colleagues at IBM's T. J. Watson Research Center in New York now show that high-performance flexible TFTs and CMOS integrated circuits can be built using carbon nanotubes ${ }^{15}$ (pictured). Their improvements in device performance are delivered through a combination of different technical developments, including an enhanced semiconductor purity and density of the nanotube films. The majority of flexible nanotube transistors to date are unipolar p-type transistors, so a doping method was also developed to fabricate n-type nanotube transistors, and deliver CMOS logic gates and integrated circuits. The IBM team was thus able to build flexible ring oscillators with stage delays as low as $5.7 \mathrm{~ns}$.

The expanding capabilities of flexible nanotube electronics is also highlighted elsewhere in this issue. In pursuit of wearable health monitors, Zhenan Bao, Boris Murmann and colleagues at Stanford University report stretchable temperaturesensing circuits made from nanotube TFTs. Notably, the circuits can suppress straindependent errors, which are often a feature of stretchable sensors, through a clever differential circuit design. As a result, the circuits maintain a temperature-sensing accuracy of $\pm 1^{\circ} \mathrm{C}$, while withstanding strains of up to $60 \%$.

Published online: 9 March 2018

https://doi.org/10.1038/s41928-018-0053-9

References

1. Tans, S. J., Verschueren, A. R. M. \& Dekker, C. Nature 393, 49-52 (1998)

2. Martel, R., Schmidt, T., Shea, H. R., Hertel, T. \& Avouris, Ph. Appl. Phys. Lett. 73, 2447-2449 (1998).

3. Iijima, S. Nature 354, 56-58 (1991).

4. Iijima, S. \& Ichihashi, T. Nature 363, 603-605 (1993).

5. Bethune, D. S. et al. Nature 363, 605-607 (1993).

6. Feder, B. J. At I.B.M., a tinier transistor outperforms its silicon cousins. The New York Times C4 (20 May 2002).

7. Bachtold, A., Hadley, P., Nakanishi, T. \& Dekker, C. Science 294, $1317-1320$ (2001).

8. Chen, Z. et al. Science 311, 1735 (2006)

9. Shulaker, M. M. et al. Nature 501, 526-530 (2013).

10. Faggin, F. Nat. Electron. 1, 88 (2018).

11. Qiu, C. et al. Science 355, 271-276 (2017).

12. Cao, Q., Tersoff, J., Farmer, D. B., Zhu, Y. \& Han, S.-J. Science 356 1369-1372 (2017).

13. Han, S.-J. et al Nat Nanotech. 12, 861-865 (2017).

14. Zhong, D. et al. Nat. Electron. 1, 40-45 (2018).

15. Tang, J. et al. Nat. Electron. https://doi.org/10.1038/s41928-0180038-8 (2018). 